\title{
Caracterización morfológica de Biotipos de Phalaris MINOR RESISTENTES Y SUSCEPTIBLE A HERBICIDAS INHIBIDORES DE LA ACCASA
}

\author{
José Luis García-Franco ${ }^{1}$, Ebandro Uscanga-MorteraA ${ }^{1,3}$, Josué Kohashi-Shibata ${ }^{1}$, \\ Antonio García-Esteva ${ }^{1}$, Petra Yáñez-Jiménez ${ }^{1}$ y Héctor Manuel Ortega-Escobar ${ }^{2}$ \\ ${ }^{1}$ Postgrado en Botánica y ${ }^{2}$ Postgrado en Hidrociencias, Colegio de Postgraduados \\ ${ }^{3}$ Autor para la correspondencia: euscanga@colpos.mx
}

\begin{abstract}
Resumen: Phalaris minor Retz. (alpistillo) es considerada como una maleza importante en el cultivo de trigo de la región del Bajío Mexicano, que ha desarrollado resistencia a herbicidas inhibidores de la Acetil Coenzima A Carboxilasa. En algunos casos se ha observado que las malezas se mimetizan con el cultivo como en algunas especies de Echinochloa con el arroz. Los objetivos del presente trabajo fueron: (1) determinar si hay diferencias morfológicas entre los biotipos resistentes y uno susceptible de $P$. minor y (2) comparar la morfología en estado vegetativo de los biotipos de $P$. minor con la del trigo. Se realizaron experimentos en Montecillo, Estado de México, en condición de no competencia en invernadero. Los biotipos resistentes empleados fueron: C4, C7, Guanajuato, Jalisco y uno susceptible. Los cultivares de trigo empleados fueron: Cortazar y Nana. Se registraron 23 variables morfológicas en todo el ciclo biológico para la comparación entre los biotipos de alpistillo y 20 en la fase vegetativa para la comparación del trigo con los biotipos de alpistillo. Los datos se analizaron mediante análisis discriminante, el cual exhibió que existen diferencias morfológicas entre los biotipos resistentes de alpistillo. Por lo anterior, es posible que la resistencia se haya desarrollado en forma independiente en los biotipos y no que la dispersión fue a partir de una población resistente inicial. Si tuvieran su origen en una sola población resistente, se esperaría que los biotipos fueran similares morfológicamente debido a su cercanía genética. El análisis discriminante también mostró una separación estadística entre los biotipos de alpistillo y los cultivares de trigo, por lo que es factible diferenciarlos visualmente en estado vegetativo.
\end{abstract}

Palabras clave: caracterización morfológica, graminicidas, resistencia a herbicidas.

\begin{abstract}
Phalaris minor Retz. (Little-seed Canary Grass) is considered an important weed of the wheat crop in the Mexican Bajio region, which has developed resistance to herbicides inhibitors of acetyl coenzyme A carboxylase. In some cases, it has been observed that weeds mimic the crop, as Echinochloa spp. with the rice. The aim of this study were: (1) to determine whether there are morphological differences between resistant and susceptible little-seed canary grass biotypes, and (2) to compare the morphology in vegetative stage of $P$. minor biotypes with the wheat. Experiments were performed in Montecillo, Estado de México, under a greenhouse and in a non-competition condition. The resistant biotypes used were: C4, C7, Guanajuato, Jalisco, and one susceptible. Wheat cultivars used were: Cortazar and Nana. Twenty morphological variables were recorded in the vegetative phase for comparison with wheat and twenty-three variables throughout the little-seed canary grass life cycle for the comparison between biotypes. Data was analyzed using discriminant analysis, which showed a statistical separation between little-seed canary grass biotypes. Thus, it is possible that the resistance had been developed independently in the biotypes and not that resistance had been developed solely from an initial population. If they were originated from a single resistant population, biotypes are expected to be similar morphologically due to its genetic closeness. The discriminant analysis also showed a statistical separation between little seed canary grass biotypes and wheat cultivars, making it practical to distinguish them visually.
\end{abstract}

Key words: graminicides, morphological characterization, resistance to herbicide.

$\boldsymbol{P}$ halaris minor Retz. (alpistillo) es una especie anual de la familia Poaceae, considerada como una maleza que afecta a cultivos de invierno en todo el mundo (Yadav y Malik, 2005). En India y en México es considerada como la principal maleza en el cultivo de trigo. Aparentemente, una de las razones de su éxito es la similitud morfológica, en su etapa vegetativa con los cereales de invierno, que propicia un control ineficiente (Afentouli y Eleftherohorinos, 1999). 
En México es la maleza más importante del cultivo de trigo en la región del Bajío, ya que reduce considerablemente el rendimiento (CESAVEG, 2007).

La problemática con esta maleza se ha acentuado con el empleo repetido de herbicidas con el mismo modo de acción, lo que ha propiciado la selección de biotipos resistentes. En la región del Bajío se tienen detectadas dos especies del género Phalaris (P. minor y $P$. paradoxa), con biotipos resistentes a herbicidas que inhiben la actividad de la Acetil Coenzima A Carboxilasa (ACCasa) (GarcíaVallejo, 2006; Heap, 2012). Cada año, se han localizado biotipos resistentes de alpistillo en sitios donde originalmente no existían, con lo cual se acentúan los problemas de producción, situación que pone en riesgo el cultivo del trigo (CESAVEG, 2007).

Para una gestión óptima de malezas, se considera necesaria la identificación morfológica correcta y oportuna. Algunas malezas poseen grandes similitudes morfológicas con los cultivos, notables ejemplos son: Avena fatua L., especies del género Lolium y Phalaris minor con el trigo; y arroz silvestre (Oryza spp.), especies del género Echinochloa y Eragrostis japonica (Thunb.) Trin. con el cultivo de arroz (Esqueda-Esquivel, 2003; Malik et al., 2003; Ortiz-Domínguez et al., 2008). Se ha estimado que en India, más de la mitad de la población de especies de Phalaris comúnmente escapa al control manual a causa de la similitud morfológica, en la fase vegetativa, con el cultivo de trigo (FAO, 1984). Sin embargo, dicha similitud también podría ser una limitante en la opción de otros métodos de control, como el químico, porque es necesaria la identificación del problema para aplicar el control. Debido a esto, es importante determinar las características morfológicas que permitan diferenciar dichas especies en estado vegetativo.

El desarrollo de la resistencia a herbicidas en especies de malezas es un proceso evolutivo que implica mutaciones en genes para la resistencia; los individuos con estos genes son seleccionados por la presión selectiva ejercida por el repetido uso de herbicidas (Gressel y Segel, 1978). En malezas, durante el proceso de desarrollo de la resistencia, se seleccionan de manera natural biotipos con características genéticas similares, por lo que las poblaciones resistentes resultantes pueden presentar características morfológicas en común y distintas a las poblaciones iniciales (Barrett, 1983; Afentouli y Eleftherohorinos, 1999; Vila-Aiub et al., 2009). $\mathrm{Si}$ los individuos resistentes en un área se originaron de un solo mutante con resistencia a herbicidas, deberían tener similitudes en sus características morfológicas. Sin embargo, si los biotipos se generaron de múltiples mutantes, deberían diferir entre sí en morfología y, por tanto, en genotipo (Tsuji et al., 2003).

Probablemente, durante el desarrollo de la resistencia, los biotipos de alpistillo se hayan diferenciado morfológicamente de las poblaciones originales como consecuencia de haberse originado de pocos individuos con esta capacidad.
Por otro lado, aun entre los individuos resistentes puede haber diferencias morfológicas si se desarrollaron en forma independiente.

En el presente estudio, se compararon las características morfológicas de alpistillo susceptible y resistente; así como entre éstos y el trigo, con los objetivos de: (1) determinar si hay diferencias morfológicas entre biotipos resistentes y uno susceptible de Phalaris minor, y (2) comparar la morfología en estado vegetativo de los biotipos de $P$. minor con la del trigo.

\section{Materiales y métodos}

Ubicación del experimento. El experimento se realizó en los Invernaderos Generales del Campus Montecillo, Colegio de Postgraduados, en Montecillo, México ( $19^{\circ} 27^{\prime} 55^{\prime \prime} \mathrm{N}, 98^{\circ}$ 54' 19" O, a una altitud de 2,250 m), durante los años 2011 y 2012.

Material vegetal. Para el estudio se utilizaron los cultivares de trigo Cortazar y Nana (usados en la región triguera de Guanajuato), un biotipo susceptible de Phalaris minor (SUSC), colectado en el estado de Guanajuato, y cuatro resistentes a herbicidas inhibidores de ACCasa, denominados Colecta 4 (C4), Colecta 7 (C7), Guanajuato (GTO), provenientes de diferentes colectas realizadas en Pénjamo, Guanajuato, y Jalisco (JAL), colectado en La Barca, Jalisco. La resistencia y susceptibilidad de los biotipos utilizados fueron previamente determinadas por trabajos realizados en la Universidad Autónoma Chapingo (García-Vallejo, 2006). Las semillas de los biotipos colectadas en campo se multiplicaron para utilizarlas en el experimento. Dicha multiplicación se realizó en invernadero, a partir del establecimiento de los biotipos colectados, donde se controló la polinización para evitar la cruza entre ellos. Una vez obtenidas, las semillas fueron guardadas en bolsas de papel encerado a temperatura ambiente hasta su utilización.

Manejo de los experimentos. Las semillas de los cultivares y biotipos se germinaron en condiciones de laboratorio, colocándolas en cajas Petri con papel filtro en el fondo y agregándoles ácido giberélico a 20 ppm para estimular la germinación (Torres-García, 2012). Las plántulas se trasplantaron después de 72 horas de iniciada la germinación a macetas de $15 \mathrm{~cm}$ de diámetro, que contenían aproximadamente $2 \mathrm{~kg}$ de suelo arcilloso como sustrato. Las plantas, así obtenidas, se establecieron en condiciones de no competencia (una planta por maceta), en un invernadero de vidrio hasta completar su desarrollo. La razón del desarrollo en condición de no competencia fue para propiciar el desarrollo en condiciones similares y uniformes. La distancia entre plantas fue de 30 $\mathrm{cm}$. La humedad del sustrato se mantuvo cercana a capacidad de campo (método gravimétrico), y el control de otras malezas se realizó en forma manual. 
Diseño experimental. El trabajo experimental se estableció en dos ocasiones, la primera se inició en septiembre de 2011 y la segunda en marzo de 2012; esto fue con el objetivo de observar la morfología y desarrollo de las plantas en condiciones ambientales contrastantes; en la primera con temperaturas medias de $15{ }^{\circ} \mathrm{C}$ y fotoperiodo de 11.5 horas luz; y en la segunda, temperaturas medias de $25^{\circ} \mathrm{C}$ y fotoperiodo de 12.5 horas luz. Se establecieron poblaciones de los cultivares de trigo y los biotipos de alpistillo a los cuales se les midieron sus características morfológicas. Dado que los resultados en ambas ocasiones manifestaron las mismas tendencias, se presentan únicamente los resultados obtenidos en 2012.

Los cultivares y biotipos fueron considerados tratamientos y se distribuyeron en un diseño completamente al azar (Oehlert, 2000) con diez repeticiones. La unidad experimental constó de una planta. Para la comparación morfológica de los biotipos y los cultivares de trigo se utilizaron los datos de la etapa vegetativa de todas las unidades experimentales. Para la comparación entre los biotipos de alpistillo se emplearon los de estos únicamente, y se consideró tanto la morfología en etapa vegetativa como reproductiva.

Para determinar el diseño del experimento se usaron como referencia trabajos acerca de caracterizaciones morfológicas en otras especies (Malik et al., 2003; Ruiz-Santaella et al., 2006; Tabacchi et al., 2006; Ortiz-Domínguez et al., 2008).

Morfología en la fase vegetativa de los biotipos de Phalaris minor resistentes, uno susceptible y cultivares de trigo. Se registraron las dimensiones de órganos cuando alcanzaron su máximo tamaño. Estas mediciones se realizaron en diferentes órganos, desde la emergencia hasta la emisión y desarrollo completo de la séptima hoja del tallo principal de las plantas; lo anterior, debido a que en los cultivares de trigo se produce la hoja bandera y la inflorescencia después de esta. Los caracteres morfológicos registrados fueron: longitud y diámetro del coleóptilo; anchura y longitud de la lámina de la primera, tercera, quinta y séptima hoja; longitud de la lígula de la tercera, quinta y séptima hoja; ángulo (porte) formado entre el tallo y la lámina de la tercera, quinta y séptima hoja; y número de culmos al aparecer la tercera, quinta y séptima hoja. Se midieron las hojas mencionadas para cubrir todo el desarrollo vegetativo, debido a que el trigo llega a producir máximo siete hojas por tallo. Además, se hicieron observaciones cualitativas de las plantas como coloración de vainas, presencia de exudado, tasa de crecimiento, presencia de aurículas, entre otras.

Caracterización morfológica de biotipos de Phalaris minor resistentes y uno susceptible. Se consideró el registro de diferentes estructuras, tanto vegetativas como reproductivas durante el desarrollo de las plantas. Además de las características antes mencionadas, en los biotipos de alpistillo se registraron: la longitud y anchura de la lámina de la novena hoja, longitud de la lígula de la novena hoja, número de culmos a la novena hoja, altura de la planta, número de hojas del culmo principal hasta la inflorescencia, anchura y longitud de la panícula y, anchura y longitud del fruto. Para esta parte del estudio no se consideró el ángulo de las hojas.

Análisis estadísticos. Se determinó la relación entre las diferentes variables de los cultivares de trigo y biotipos de Phalaris minor mediante el uso de análisis multivariado, utilizando la técnica de análisis discriminante. Este análisis se realizó con el paquete Statistical Analysis System 9.1 (SAS, 2002) y el programa de distribución libre Past 3.0 (Hammer et al., 2001).

\section{Resultados}

Morfología en fase vegetativa de los biotipos de Phalaris minor susceptible y resistentes, y cultivares de trigo. En el cuadro 1 se enlistan las características generales en las cuales el alpistillo y el trigo difieren. Los biotipos de P. minor, tanto el susceptible como los resistentes, presentaron diferencias respecto a los cultivares de trigo Nana y Cortazar durante la fase vegetativa.

Con el análisis discriminante, la formación de funciones discriminantes (FD) y el cálculo de distancias de Mahalanobis, se demostró la diferencia estadística entre los cultivares y los biotipos de alpistillo y su separación en grupos independientes (Cuadro 2). Lo anterior debido a las diferencias en dimensiones de los órganos. Las variables morfológicas de mayor correlación con la FD1 y que son determinantes en la separación de los biotipos de alpistillo de los cultivares de trigo son: diámetro de coleóptilo, anchura de primera y séptima lámina foliar, longitud de tercera lámina foliar y longitud de lígula de quinta hoja (Cuadro 3).

Los biotipos de alpistillo produjeron láminas foliares de tamaño similar a las del trigo a partir de la quinta hoja. Cuando esto sucedió, otras características tales como el tamaño de la lígula, el ángulo de la lámina foliar y la cantidad de culmos por planta, adquirieron relevancia para la separación estadística entre ellos. En la fase vegetativa, los biotipos de

Cuadro 1. Diferencias morfológicas en la fase vegetativa entre trigo y alpistillo (Yadav y Malik, 2005). TC: tasa de crecimiento

\begin{tabular}{lcc}
\hline Característica & Alpistillo & Trigo \\
\hline nervadura de la vaina & rosa púrpura & verde \\
longitud de lígula & $7 \mathrm{~mm}$ & $2 \mathrm{~mm}$ \\
aurícula & ausente & presente \\
crecimiento inicial & lento: $\mathrm{TC}=1.25 \mathrm{~g} \mathrm{~d}^{-2}$ & rápido: $\mathrm{TC}=6.58 \mathrm{~g} \mathrm{~d}^{-2}$ \\
(hasta la tercer hoja) & en la base y en la & en la base \\
ramificación & parte superior & \\
exudado (savia) & rosa & transparente \\
\hline
\end{tabular}


José Luis García-Franco et aL.

Cuadro 2. Probabilidad de igualdad entre los biotipos mediante la comparación de la distancia de Mahalanobis con la distancia resultante del análisis discriminante de las características morfológicas vegetativas de los biotipos y cultivares de trigo desarrollados en invierno. C7: Colecta 7, C4: Colecta 4, GTO: Guanajuato, JAL: Jalisco, SUSC: Susceptible. Valores con * indican diferencia estadística entre los biotipos y cultivares interceptados.

\begin{tabular}{|c|c|c|c|c|c|c|c|}
\hline \multirow{2}{*}{$\begin{array}{l}\text { Biotipos y } \\
\text { cultivares }\end{array}$} & \multicolumn{5}{|c|}{ Biotipos } & \multicolumn{2}{|c|}{ Cultivares } \\
\hline & $\mathrm{C} 7$ & C4 & GTO & JAL & SUSC & CORTAZAR & NANA \\
\hline C7 & 1.0000 & $<.0001^{*}$ & $<.0001^{*}$ & $<.0001^{*}$ & $<.0001^{*}$ & $<.0001^{*}$ & $<.0001^{*}$ \\
\hline $\mathrm{C} 4$ & & 1.0000 & $0.0010^{*}$ & 0.0075 & 0.0241 & $<.0001^{*}$ & $<.0001^{*}$ \\
\hline GTO & & & 1.0000 & $0.0002 *$ & 0.0034 & $<.0001^{*}$ & $<.0001 *$ \\
\hline$J A L$ & & & & 1.0000 & 0.0918 & $<.0001^{*}$ & $<.0001 *$ \\
\hline SUSC & & & & & 1.0000 & $<.0001^{*}$ & $<.0001^{*}$ \\
\hline CORTAZAR & & & & & & 1.0000 & $<.0001 *$ \\
\hline NANA & & & & & & & 1.0000 \\
\hline
\end{tabular}

alpistillo presentaron hasta 13 culmos por planta, en comparación a los dos o tres producidos por el trigo. La longitud de la lígula en los biotipos fue tres veces más grande que la del trigo. El ángulo de las láminas foliares fue en promedio de $90^{\circ}$ en los biotipos, mientras que en el trigo fue de $30^{\circ}$.

Aun entre algunos biotipos se tuvieron diferencias; la C7

Cuadro 3. Estructura de las funciones discriminantes 1 y 2 obtenidas en el análisis estadístico aplicado a las características morfológicas vegetativas de los biotipos de alpistillo y cultivares de trigo desarrollados en invierno. FD1: función discriminante 1, FD2: función discriminante 2. Valores con * indican variables de mayor magnitud asociadas a su función discriminante correspondiente.

\begin{tabular}{lcc}
\hline Variable original & FD1 & FD2 \\
\hline diámetro de coleóptilo & $0.930994^{*}$ & 0.035638 \\
longitud del coleóptilo & -0.264838 & -0.038202 \\
longitud de primera lámina foliar & -0.235602 & $-1.061860^{*}$ \\
anchura de primera lámina foliar & $5.480755^{*}$ & 1.908582 \\
ángulo de primera lámina foliar & 0.072105 & -0.283175 \\
longitud de tercera lámina foliar & $1.398303^{*}$ & -0.295462 \\
anchura de tercera lámina foliar & -0.45924 & 0.901550 \\
longitud de lígula de tercera hoja & -0.179531 & -0.283228 \\
ángulo de tercera lámina foliar & -0.268811 & -0.017353 \\
número de culmos a tercera hoja & -0.600385 & 0.613690 \\
longitud de quinta lámina foliar & -0.490047 & $-0.948068^{*}$ \\
anchura de quinta lámina foliar & 0.473250 & -0.098211 \\
longitud de lígula de quinta hoja & $-0.994128^{*}$ & -1.112054 \\
ángulo de quinta lámina foliar & -0.147265 & 0.475739 \\
número de culmos a quinta hoja & 0.337773 & -0.241429 \\
longitud de séptima lámina foliar & -0.565674 & $0.979714^{*}$ \\
anchura de séptima lámina foliar & $0.964112^{*}$ & 0.022900 \\
longitud de lígula de séptima hoja & -0.759359 & -0.149947 \\
ángulo de séptima lámina foliar & -0.139431 & 0.185954 \\
número de culmos a séptima hoja & -0.031161 & $1.74683 *$ \\
\hline
\end{tabular}

se separó del resto; la C4 se separó del GTO, y el GTO del JAL (Cuadro 2). La distancia mayor entre los biotipos de alpistillo estuvo dada por la FD2. Las características morfológicas que mayor influencia tuvieron en esta función fueron: número de culmos a séptima hoja y la longitud de primera, quinta y séptima lámina foliar. La separación entre algunos biotipos no es totalmente clara, debido a que existe sobreposición entre algunos individuos de estos biotipos al momento de graficar las primeras dos FD (Figura 1).

\section{Caracterización morfológica de biotipos de Phalaris minor resistentes y susceptible.}

Colecta 4.- Plantas de porte medio, de 85 a $100 \mathrm{~cm}$ de altura. Crecimiento en ocasiones decumbente. Lámina foliar de hasta $35 \mathrm{~cm}$ de largo y hasta $1 \mathrm{~cm}$ de ancho; el número de hojas sobre el culmo principal de hasta 17; la longitud de la panícula aproximadamente de $5.6 \mathrm{~cm}$. El número de frutos por inflorescencia de 270 a 280 ; el tamaño del fruto de 2.6 $\mathrm{mm}$ de largo y $1.3 \mathrm{~mm}$ de ancho. El ancho de las hojas es mayor que los demás biotipos y presenta pocos culmos.

Colecta 7.- Plantas de porte bajo, de 79 a $85 \mathrm{~cm}$ de altura. Crecimiento en ocasiones decumbente. Lámina foliar de $29 \mathrm{~cm}$ de largo y hasta $8 \mathrm{~mm}$ de ancho; el número de hojas sobre el culmo principal puede llegar hasta 17 en promedio; en condiciones de no competencia hasta 19 culmos; panícula aproximadamente de $5.6 \mathrm{~cm}$ de longitud. El número de frutos por inflorescencia de 275 a 280; el tamaño del fruto de $2.6 \mathrm{~mm}$ de largo y $1.3 \mathrm{~mm}$ de ancho. En la mayoría de sus características es similar al Susceptible. Se caracteriza por tener hojas angostas y erectas, un porte bajo y gran cantidad de culmos.

Guanajuato.- Plantas de porte medio, de 87 a $110 \mathrm{~cm}$ de altura. Crecimiento en ocasiones decumbente, en promedio, 10 culmos en condiciones de no competencia. Lámina foliar de $40 \mathrm{~cm}$ de largo y hasta $1.1 \mathrm{~cm}$ de ancho; el número de hojas sobre el culmo principal 17 en promedio; el número de panículas por planta es bajo; panícula de $6.7 \mathrm{~cm}$ de longitud. El número de frutos por inflorescencia de 293; fruto de tamaño medio, de $2.6 \mathrm{~mm}$ de largo y $1.3 \mathrm{~mm}$ de ancho. En 


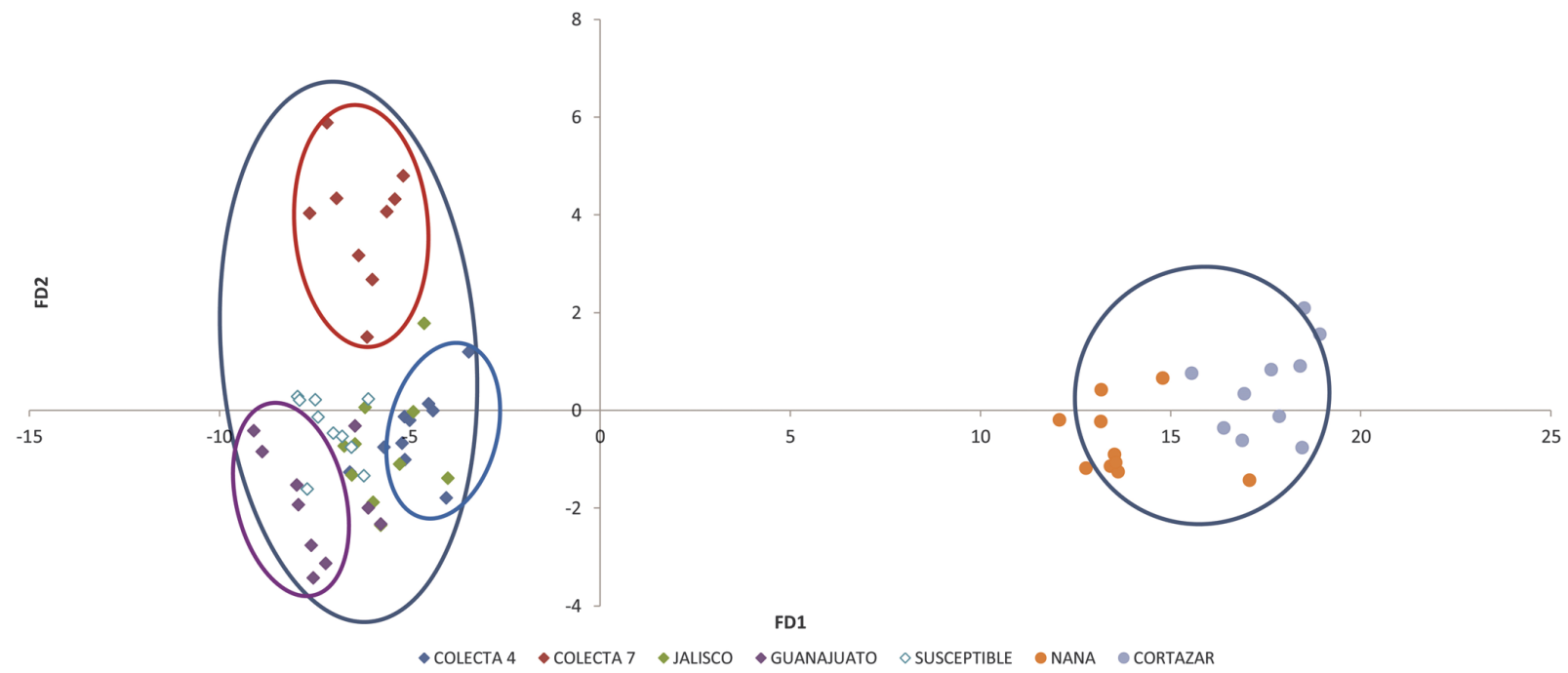

Figura 1. Ordenación de las observaciones en un plano ortogonal definido por las funciones discriminantes 1 y 2 (FD1 y FD2) de las características morfológicas vegetativas de los biotipos de alpistillo y cultivares de trigo desarrollados en invierno. Las elipses indican el intervalo de confianza del $95 \%$ para los cultivares y biotipos.

la mayoría de sus características, el Guanajuato es similar a la Colecta 4. Presenta hojas anchas con apariencia vigorosa y pocos culmos.

Jalisco.- Plantas de porte alto, de 87 a $115 \mathrm{~cm}$ de altura. Crecimiento erecto. En condiciones de no competencia hasta 14 culmos. Lámina foliar de $27 \mathrm{~cm}$ de largo y hasta $7 \mathrm{~mm}$ de ancho, además, las hojas son erectas y pocas veces laxas; el número de hojas sobre el culmo principal de hasta 21; el número de panículas por planta en promedio 14; panícula aproximadamente $5.6 \mathrm{~cm}$ de longitud; el número de frutos por inflorescencia de 270; fruto de tamaño grande, de 2.9 $\mathrm{mm}$ de largo y $1.5 \mathrm{~mm}$ de ancho. Se diferencia por tener hojas angostas, cortas y un porte totalmente erecto.

Susceptible.- Plantas de porte bajo, de 82 a $110 \mathrm{~cm}$ de altura. Crecimiento en ocasiones decumbente. En condiciones de no competencia hasta 21 culmos. La lámina foliar de $30 \mathrm{~cm}$ de largo y hasta de $8.5 \mathrm{~mm}$ de ancho; en promedio, el número de hojas sobre el culmo principal es de 20; panícula de aproximadamente $5.6 \mathrm{~cm}$ de longitud. El número de frutos por inflorescencia de aproximadamente 317; fruto de tamaño medio, de $2.6 \mathrm{~mm}$ de largo y $1.3 \mathrm{~mm}$ de ancho. En la mayoría de sus características es similar a la Colecta 7.

Mediante el análisis discriminante, se determinó que existen diferencias estadísticas entre los biotipos, excepto entre el JAL y SUSC (Cuadro 4). En la representación gráfica de las primeras dos FD en un plano ortogonal, se observa que aunque hubo diferencias estadísticas entre los biotipos, existe poca distancia entre algunos de ellos, lo que indica cierta relación debido a su origen común. Los biotipos GTO y C4 se encuentran más relacionados entre sí que con el resto de biotipos; de igual forma JAL y SUSC presentaron ciertas similitudes que los acercan (Figura 2).

Las variables detectadas en el análisis discriminante con mayor relevancia, y por tanto, asociadas a la variabilidad entre los biotipos son: longitud de la quinta y séptima lámina foliar, altura de la planta y número de hojas por culmo (Cuadro 5). La C7 presenta hojas más largas que el resto de biotipos, pero menor altura y número de hojas. GTO y $\mathrm{C} 4$ presentaron una altura media y mayor anchura de láminas foliares que el resto de biotipos. JAL y SUSC se diferenciaron por tener una mayor altura y número de hojas en el culmo principal.

Al analizar los resultados obtenidos se encontró que las características responsables de la mayor diversidad entre los biotipos son constantes. Los caracteres más relevantes fueron: ancho y largo de quinta, séptima y novena lámina foliar; número de hojas por culmo y altura de la planta (Cuadro 6). Mediante las variables estudiadas se demostró que cada biotipo es diferente, sólo en algunos casos existe una reducida sobreposición entre ellos. Además, por las diferencias en las distancias calculadas entre los biotipos (Cuadro 4), es posible separar los biotipos y establecer relaciones de similitud morfológica entre el GTO y C4.

Cuadro 4. Probabilidad de igualdad entre los biotipos mediante la comparación de la distancia de Mahalanobis con la distancia cuadrada resultante del análisis discriminante de las características morfológicas de los biotipos de alpistillo desarrollados en invierno. C7: Colecta 7, C4: Colecta 4, GTO: Guanajuato, JAL: Jalisco, SUSC: Susceptible. Valores con * indican diferencia estadística entre los biotipos interceptados.

\begin{tabular}{llllll}
\hline Biotipos & C7 & C4 & GTO & JAL & SUSC \\
\hline C7 & 1.0000 & $0.0004^{*}$ & $<.0001^{*}$ & $<.0001^{*}$ & $<.0001^{*}$ \\
C4 & & 1.0000 & $0.0012^{*}$ & $0.0022^{*}$ & $<.0001^{*}$ \\
GTO & & & 1.0000 & $0.0001^{*}$ & $0.0002^{*}$ \\
JAL & & & & 1.0000 & 0.0273 \\
SUSC & & & & & 1.0000
\end{tabular}




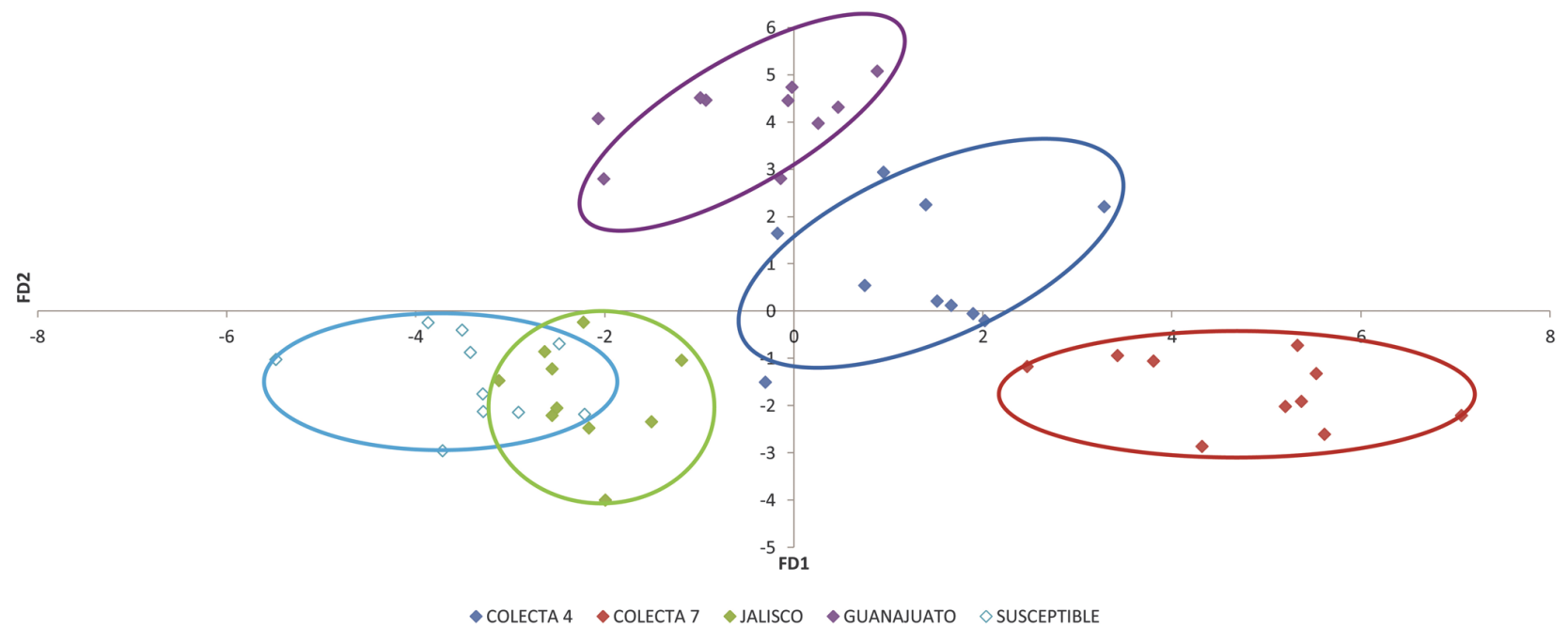

Figura 2. Ordenación de las observaciones en un plano ortogonal definido por las funciones discriminantes 1 y 2 (FD1 y FD2) de las características morfológicas de los biotipos de alpistillo desarrollados en invierno. Las elipses indican el intervalo de confianza del $95 \%$ para los biotipos.

Cuadro 5. Estructura de las funciones discriminantes 1 y 2 obtenidas en el análisis estadístico aplicado a las características morfológicas de los biotipos de alpistillo desarrollados en invierno. FD1: función discriminante 1, FD2: función discriminante 2. Valores con * indican variables de mayor magnitud asociadas a su función discriminante correspondiente.

\begin{tabular}{lcc}
\hline Variable original & FD1 & FD2 \\
\hline diámetro de coleóptilo & -0.507820 & 0.247044 \\
longitud de coleóptilo & 0.437202 & -0.322280 \\
longitud de primera lámina foliar & -0.731532 & -0.018703 \\
anchura de primera lámina foliar & 0.674554 & -0.796135 \\
longitud de tercera lámina foliar & -0.650646 & 0.302536 \\
anchura de tercera lámina foliar & 1.000726 & 0.073129 \\
longitud de lígula de tercera hoja & -0.351212 & -0.300254 \\
longitud de quinta lámina foliar & $-1.217899^{*}$ & -0.348456 \\
anchura de quinta lámina foliar & 1.188150 & -0.101223 \\
longitud de lígula de quinta hoja & -0.229996 & 0.511414 \\
longitud de séptima lámina foliar & $1.147326 *$ & 0.777341 \\
anchura de séptima lámina foliar & -0.432956 & 0.437927 \\
longitud de lígula de séptima hoja & -0.452770 & -0.030534 \\
longitud de novena lámina foliar & 0.3163434 & $-1.071402 *$ \\
anchura de novena lámina foliar & 0.117704 & 0.546518 \\
longitud de lígula de novena hoja & -0.369410 & -0.117519 \\
número de culmos a la novena hoja & -0.053213 & -0.642296 \\
altura de la planta & $-1.420857 *$ & -0.119930 \\
número de hojas por culmo & $-1.496727 *$ & -0.821298 \\
longitud de panícula & 0.595376 & $1.113552 *$ \\
anchura de panícula & -0.137242 & -0.177482 \\
anchura del fruto & 0.551972 & -0.147276 \\
longitud del fruto & -0.340551 & -0.322708 \\
\hline
\end{tabular}

\section{Discusión}

Morfología en fase vegetativa de los biotipos de Phalaris minor resistentes, uno susceptible y cultivares de trigo. $\mathrm{Al}$ inicio del crecimiento, tanto el ancho y largo del coleóptilo y la primera lámina foliar, son de mayores dimensiones en los cultivares de trigo que en el alpistillo. Lo anterior parece estar relacionado con el tamaño de la semilla de las dos especies, ya que la del trigo es de $9 \mathrm{~mm}$ de largo en comparación con la del alpistillo que es de $3 \mathrm{~mm}$. Por lo tanto, el tamaño del embrión y el endospermo es mayor, lo que le confiere una ventaja inicial en el crecimiento cuando las condiciones son idóneas para reanudar dicho crecimiento.

Aunque existen diferencias entre las características del alpistillo y el trigo, el parecido le confiere posibilidades de escape al control manual y químico. Dicho escape puede ocurrir aun cuando las personas responsables de la gestión de malezas tengan experiencia en su identificación (FAO, 1984; Tominaga y Yamasue, 2004; Radosevich et al., 2007). Barrett (1983) destacó la importancia de la identificación correcta de los biotipos durante la gestión de las malezas.

Especies del género Echinochloa han sido estudiadas con el propósito de observar la similitud morfológica con el cultivo de arroz (Ruiz-Santaella et al., 2006); los resultados de estas investigaciones separaron estadísticamente las especies. En el caso de los biotipos de E. crus-galli, se ha relacionado el grado de susceptibilidad a herbicidas con la morfología, además de observar las similitudes con el arroz (Malik et al., 2003).

En el alpistillo, la similitud en la morfología con la del de trigo en la fase vegetativa no tiene relación con la resistencia a herbicidas, ya que si así fuera, se esperaría mayor parecido con los biotipos resistentes y no fue así. El parecido es debido a la estrecha relación que tiene esta maleza 
Cuadro 6. Valores promedio $(\mathrm{mm})$ de los caracteres morfológicos medidos en los biotipos de Phalaris minor desarrollados en invierno. Iqh: longitud de quinta lámina foliar, aqh: anchura de quinta lámina foliar, Ish: longitud de séptima lámina foliar, ash: anchura de séptima lámina foliar, Inh: longitud de novena lámina foliar, anh: anchura de novena lámina foliar, nh: número de hojas por culmo, hp: altura de la planta.

\begin{tabular}{lccccc}
\hline Caracteres & C4 & C7 & JAL & GTO & SUSC \\
\hline Iqh & $245.7 \pm 10.33$ & $210.4 \pm 6.69$ & $216.5 \pm 6.05$ & $258.3 \pm 9.40$ & $202.4 \pm .54$ \\
aqh & $7.6 \pm 0.25$ & $6.6 \pm 0.25$ & $7.1 \pm 0.26$ & $7.6 \pm 0.30$ & $6.7 \pm 0.22$ \\
Ish & $290.2 \pm 11.34$ & $282.2 \pm 10.90$ & $275.5 \pm 7.92$ & $320.5 \pm 15.4$ & $246.3 \pm 12.78$ \\
ash & $8.5 \pm 0.30$ & $7.6 \pm 0.22$ & $7.5 \pm 0.30$ & $9.1 \pm 0.31$ & $7.3 \pm 0.38$ \\
Inh & $332.3 \pm 17.89$ & $359.9 \pm 16.59$ & $366.6 \pm 13.89$ & $407.2 \pm 17.22$ & $332.2 \pm 14.76$ \\
anh & $9.3 \pm 0.49$ & $9.13 \pm 0.45$ & $9.1 \pm 0.32$ & $11.5 \pm 0.57$ & $8.4 \pm 0.38$ \\
nh & $17.6 \pm 0.56$ & $17.4 \pm 0.33$ & $20.6 \pm 0.37$ & $17.6 \pm 0.58$ & $19.9 \pm 0.1$ \\
hp & $1,008.4 \pm 29.82$ & $840.8 \pm 47.44$ & $1,058.4 \pm 19.47$ & $1,162.7 \pm 43.6$ & $1,130.4 \pm 23.94$ \\
\hline
\end{tabular}

con los cereales (Barrett, 1983). Aun así, con la observación detallada es posible distinguir la maleza del cultivo.

Caracterización morfológica de biotipos de Phalaris minor resistentes y susceptible. Con base a los resultados obtenidos no es posible aseverar que los biotipos de alpistillo resistentes a herbicidas son diferentes al Susceptible, debido a que no existe una separación que así lo muestre, ni que la morfología esté ligada a la resistencia. Además, para llegar a ese tipo de conclusiones se necesitaría una mayor cantidad de colectas susceptibles (mismo número que resistentes), para así poder establecer la relación entre la morfología y el grado de susceptibilidad.

Con los resultados obtenidos podemos inferir las relaciones en torno al proceso de desarrollo de la resistencia de los biotipos y la morfología. En Echinochloa, Tsuji et al. (2003) dedujeron que debido a la similitud de la morfología (ayudado de técnicas moleculares para su corroboración), el origen de la resistencia ocurrió en un área y a partir de ahí tuvo lugar la dispersión; esto debido a que la similitud entre los biotipos que estudiaron fue alta. En el caso de los biotipos de Phalaris minor resistentes a herbicidas inhibidores de la ACCasa, la resistencia posiblemente se desarrolló de forma independiente, ya que si el origen fuera una sola población resistente que se dispersó, se esperaría que todos los biotipos tuvieran gran similitud morfológica debido al corto tiempo que ha pasado a partir de su detección, y no fue así.

La caracterización morfológica de poblaciones o biotipos es un instrumento para establecer relaciones genéticas, y por lo tanto evolutivas. Con el apoyo de técnicas de biología molecular es posible corroborar y complementar esta información. Torres-García (2012) realizó la secuenciación del gen ACCasa en los biotipos resistentes y el susceptible utilizados en esta investigación, y demostró que las mutaciones en este gen que confieren la resistencia a herbicidas son diferentes en cada biotipo. Estos resultados refuerzan los obtenidos en la presente investigación donde, con las características morfológicas, se estableció la diferencia entre los biotipos, aunque las semillas de los biotipos se colectaron en una misma región.
Los trabajos morfológicos de malezas en algunos casos se han reforzado al apoyarse con técnicas de biología molecular. Tabacchi et al. (2006) y Damalas et al. (2008) llevaron a cabo trabajos donde complementan la caracterización morfología con técnicas genéticas. Ellos estudiaron especies del género Echinochloa y demostraron la relacion entre estas. Además, Damalas et al. (2008) hacen inferencia sobre la relación de las características con la susceptibilidad a herbicidas.

En algunos otros estudios morfológicos, las diferencias morfológicas y fisiológicas se deben al origen geográfico o ecológico de las poblaciones (Hübner et al., 2003), y a esto también se atribuye el grado de sensibilidad a los herbicidas. En el presente estudio no es posible afirmar que pase lo mismo, ya que los biotipos provienen de áreas ubicadas en la misma región y un ambiente similar.

Por los resultados y lo antes mencionado, se concluye que existen diferencias morfológicas en la etapa vegetativa entre los biotipos de alpistillo y los cultivares de trigo que hacen posible su identificación. Además, es muy probable que las encontradas tanto en los biotipos resistentes a herbicidas entre sí y con respecto al susceptible, a pesar de que provienen de una misma región, se deban a que cada biotipo desarrolló la resistencia de forma independiente.

\section{Agradecimientos}

A Antonio Tafoya Razo, profesor-investigador del Departamento de Parasitología de la Universidad Autónoma Chapingo por proporcionar las semillas de los biotipos empleados. A Eduardo Villaseñor Mir, investigador del Instituto de Investigaciones Forestales Agrícolas y Pecuarias (INIFAP), Campo Experimental Valle de México, por proporcionar los cultivares de trigo utilizados y a los revisores anónimos que contribuyeron a mejorar este manuscrito.

\section{Literatura citada}

Afentouli C.G. y Eleftherohorinos I.G. 1999. Competition between wheat and canarygrass biotypes and their response to herbicides. Weed Science 47:55-61. 
Barrett S. H. 1983. Crop mimicry in weeds. Economic Botany 37:255-282.

CESAVEG. Comité Estatal de Sanidad Vegetal de Guanajuato. 2007. Campaña de manejo fitosanitario de trigo. Manejo integrado de malezas. Folleto Técnico. Secretaría de Agricultura, Ganadería, Desarrollo rural, Pesca y Alimentación. Irapuato.

Damalas C.A., Dhima K.V. y Eleftherohorinos I.G. 2008. Morphological and physiological variation among species of the genus Echinochloa in Northern Greece. Weed Science 56:416-423

Esqueda-Esquivel V.A. 2003. Morfología y crecimiento de cuatro biotipos de arroz rojo y de la variedad Milagro Filipino. Agricultura Técnica en México 29:35-47.

FAO. Food and Agriculture Organization. 1984. Improving weed management. FAO plant production and protection papers. Roma.

García-Vallejo F. 2006. Resistencia de cuatro biotipos de Phalaris minor Retz. a los herbicidas clodinafop propargyl y tralkoxidim. Tesis de licenciatura. Departamento de Parasitología Agrícola, Universidad Autónoma Chapingo. Chapingo. 74 pp.

Gressel J. y Segel L.A. 1978. The paucity of plants evolving genetic resistance to herbicides: possible reasons and implications. Journal Theoretical Biology 75:349-371.

Hammer O., Harper D.A.T. y Ryan P.D. 2001. PAST: Paleontological Statistics software package for education and data analysis. Paleontologia Electronica.

Heap I. 2012. The International Survey of Herbicide Resistant Weeds. <www.weedscience.com> (consultado 1 marzo 2013).

Hübner R., Fykse H., Hurle K. y Klemsdal S.S. 2003. Morphological differences, molecular characterization, and herbicide sensitivity of catchweed bedstraw (Galium aparine) populations. Weed Science 51:214-225.

Malik M.S., Talbert R.E., Burgos N.R., Ottis B.V. y Ellis A.T. 2003. Characterization of herbicide resistant biotypes of barnyardgrass. AAES Research Series 517:116-121.

Oehlert G.W. 2000. A first course in design and analysis of experiments. W.H. Freeman and Company. Nueva York.

Ortiz-Domínguez A., Miranda R., Figueroa R. y Ramis C. 2008. Caracterización morfológica de una población F2 obtenida del cruce natural entre un cultivar arroz y un arroz rojo (parte I). Agronomía Tropical 58:299-307.

Radosevich S.R., Holt J.S. y Ghersa C.M. 2007. Ecology of Weeds and Invasive Plants: Relationship to Agriculture and Natural Resource Management. $3^{\text {a }}$ ed. John Wiley \& Sons, Inc., Hoboken.

Ruiz-Santaella J.P., Bastida F., Menéndez J. y Prado R. de. 2006. Caracterización morfológica de poblaciones de 6 especies de Echinochloa con diferente grado de susceptibilidad a cihalofop butilo. En: Mondragón P.G., Domínguez V. J.A., Martínez D.G. y Ocampo RR.A. Eds. Memoria XVI Congreso Latinoamericano de Malezas y XXIV Congreso Nacional de la Ciencia de la Maleza, pp. 549-553, Asociación Latinoamericana de Malezas, Manzanillo.

SAS. Statistical Analysis System. 2002. Versión 9.1. SAS Institute, Inc. Cary.

Tabacchi M., Mantegazza R., Spada A. y Ferrero A. 2006. Morphological traits and molecular markers for classification of Echinochloa species from Italian rice fields. Weed Science 54:1086-1093.

Tominaga T. y Yamasue Y. 2004. Crop-associated weeds: The strategy for adaptation. En: Inderjit Ed. Weed Biology and Management, pp 47-63, Kluwer Academic Publishers. Norwell.

Torres-García J.R. 2012. Diferencias en la aptitud entre colectas de "alpistillo" (Phalaris minor Retz.) Resistentes y Susceptible a inhibidores de la ACCasa. Tesis doctoral, Postgrado de Botánica, Colegio de Postgraduados Campus, Montecillo, Texcoco. 138 pp.

Tsuji R., Fischer A.J., Yoshino M., Roel A., Hill J.E. y Yamasue Y. 2003. Herbicide-resistant late watergrass (Echinochloa phyllopogon): similarity in morphological and amplified fragment length polymorphism traits. Weed Science 51:740-747.

Vila-Aiub M.M., Neve P. y Powles S.B. 2009. Fitness costs associated with evolved herbicide resistance alleles in plants. New Phytologist 184:751-767.

Yadav A. y Malik R.K. 2005. Herbicide resistant Phalaris minor in wheat - a sustainability issue. Department of Agronomy and Directorate of Extension Education, Chaudhary Charan Singh Haryana Agricultural University, Hisar.

Recibido: 3 de marzo de 2013

Aceptado: 5 de julio de 2013 